Supporting information for:

\title{
Remote Loading of Gas Bubbles into Polylactic Acid Microcapsules Creates Acoustically Active Janus Particles
}

Arvin Honari, Pallavi S. Kapilavaih, Nasrin Akter, Shashank R. Sirsi*

Department of Bioengineering, Erik Jonsson School of Engineering, The University of Texas at Dallas, Richardson, Texas, USA, 75080

*Shashank.Sirsi@UTDallas.edu

This document Includes:

1. Materials and Methods

2. Supplementary Figures

3. Supplementary Videos

\section{Materials and Methods}

\section{Materials}

Poly(L-lactic acid), IV $1.8 \mathrm{dl} / \mathrm{g}$ from Polysciences. Dichloromethane from Sigma-Aldrich. Probe tip sonicator from Misonics, ultrasonic liquid processors. Poly (vinyl alcohol), MW 25000, 88\% hydrolyzed from Polysciences. Homogenizer PT 3100 Polytron. Isopropanol alcohol from SigmaAldrich. Sorvall ST 8 centrifuge from Thermo Fisher Scientific (Germany) and benchtop centrifuge from Sprout, Heathrow Scientific, Vernon Hills, IL, USA. Hexane from Sigma-Aldrich. Propane-1,2,3triol (glycerol, 92.09 FW) from Fisher bioreagents. Propane-1,2-diol (propylene glycol, 76.1 FW) from Fisher Chemicals. Maxi Mix II Thermolyne vortex. Multisizer 4e Coulter Counter (MS4, from Beckman Coulter, Brea, CA). Glass coverslip from Fisher Scientific, Waltham, MA, USA. Perfusion chamber $1550 \mu \mathrm{L}$ from Electron microscopy science. Calcein from MP Biomedicals, LLC, Solon, $\mathrm{OH}$, USA. BX50 Upright Microscope ACH 60X/0.80 $\infty / 0.17$ objective from Olympus. Scanning Electron Microscopy (Zeiss SUPRA 40 SEM). Ultrasound transducer (H-131 from Sonic Concepts, $1.1 \mathrm{MHz}$, Bothell, WA, USA). Hydrophone (Y-107 from Sonic Concepts, Bothell, WA, USA). Plate reader (Synergy H4 from Biotek, Winooski, VT, USA). Olympus 1X-71 inverted microscope (ACH 60X/0.80 $\infty / 0.17$ objective)

\section{Methods}

\section{Preparation of Polylactic acid Microcapsules}

A double emulsion technique similar to the method previously established in the literature was used to create polylactic acid microcapsules ${ }^{1}$. PLA was dissolved in dichloromethane for $1 \mathrm{~h}$. Phosphate buffered saline (1XPBS) was added to the solution and sonicated for $20 \mathrm{~s}$ using a probe tip sonicator to create a water in oil (W/O) emulsion, while the sample was kept cool in an ice bath. The solution was slowly added to polyvinyl alcohol (PVA) at $4^{\circ} \mathrm{C}$ and then homogenized for $10 \mathrm{~min}$ at $9500 \mathrm{rpm}$ to produce the second emulsion (W/O/W). The solution was added to isopropanol alcohol and was left under the hood overnight while mixing using a magnetic stir bar. On day two, three centrifugations 
were performed at $3200 \times \mathrm{g}$ for $8 \mathrm{~min}$ to separate the microcapsules from unincorporated PLA polymers. The separated microcapsules were washed with hexane three times. After performing the hexane wash, the solution was left under the hood overnight to fully evaporate the hexane from the solution. The resulting PLA microcapsule sample was stored at $4^{\circ} \mathrm{C}$.

\section{Air Nucleation in Polylactic acid Microcapsules}

For nucleating gas bubbles in PLA microcapsules and producing acoustic vesicles, a fixed concentration of microcapsules was hydrated with a mixture of propylene glycol (PPG), glycerol (1:1 ratio), and 1X PBS. The solution was then vortexed for $5 \mathrm{~s}$. Different concentrations of propylene glycol and glycerol $(10 \% \mathrm{v} / \mathrm{v}, 20 \% \mathrm{v} / \mathrm{v}, 30 \% \mathrm{v} / \mathrm{v}$, and $40 \% \mathrm{v} / \mathrm{v})$ were used to see the effect of supersaturation on the degree of nucleation. Microscopy images were acquired to compare the degree of air nucleation in each sample. To further confirm the nucleation hypothesis, the production of acoustic vesicles was also attempted using deionized water and ethanol. Microcapsules were suspended in different concentrations of ethanol (25\% v/v, $50 \% \mathrm{v} / \mathrm{v}, 75 \% \mathrm{v} / \mathrm{v}$, and $100 \% \mathrm{v} / \mathrm{v})$ and deionized water was added to the microcapsules followed by $5 \mathrm{~s}$ of vortex mixing. Microscopy images were acquired from all samples.

\section{Characterization of Acoustic Vesicles Using Microscopy}

Acoustic vesicles were visualized by brightfield microscopy with a BX50 Upright Microscope (ACH $60 \mathrm{X} / 0.80 \propto / 0.17$ objective). Acoustic vesicles were put onto a $25 \times 75 \times 1 \mathrm{~mm}$ microscope slide and placed under a glass coverslip. Microscopy images were taken by a Rolera Bolt CMOS QImaging Camera (Surrey, BC, Canada). Images from the coverslip were acquired to visualize buoyant acoustic vesicles and from the microscope slide to see heavier unloaded microcapsules that will naturally sink to the microscope slide. To quantify the nucleation events, the number of nucleated bubbles from the coverslip were counted using NIH ImageJ software. The counting was done by averaging eight random images from each sample $(\mathrm{N}=3)$. ANOVA and post hoc t-test was done on the dataset to measure significance.

\section{Nucleation of Acoustic Vesicles in Agarose Gel}

Microcapsules were embedded in 1\% agarose gel. A small block of solidified gel was placed inside of a perfusion chamber and the perfusion chamber was placed under the upright microscope. A mixture of $30 \%$ propylene glycol (v/v) and 30\% glycerol (v/v) 1XPBS was perfused in the chamber to cover microcapsule-embedded agarose gel. 2-hour time-lapse videos were taken to visualize the nucleation process in the PLA microcapsules when the PPG and glycerol mixture was slowly perfused through the gel block. As a negative control, 1X PBS was added to the microcapsule-loaded agarose gel, and videos were taken for the same period. ImageJ software was used to analyze the videos.

\section{Calcein Loading on Acoustic Polylactic acid Vesicles}

For loading calcein in acoustic vesicles, the same protocol as making regular acoustic vesicles was used. However, instead of using water for the first emulsion, $50 \mathrm{mM}$ calcein solution was used and the protocol for making microcapsules and acoustic vesicles was followed.

\section{Separation of Acoustic Vesicles}

To separate buoyant acoustic vesicles from non-buoyant microcapsules, the acoustic vesicles were placed in a $2 \mathrm{~mL}$ centrifuge tube right after nucleation. 8 centrifugations were performed at $1500 \times \mathrm{g}$ 
for $1 \mathrm{~min}$. After each centrifugation, the supernatant of the acoustic vesicle solution was collected in a $10 \mathrm{~mL}$ syringe and fresh 1 XPBS was added to the pellet, followed by a quick vortex mix. After the last centrifugation, the pellet was also collected for microscopy characterization. The $10 \mathrm{~mL}$ syringe went through 3 additional centrifugations at $300 \times$ g for $3 \mathrm{~min}$ to separate buoyant acoustic vesicles. The separated acoustic vesicles were characterized by brightfield microscopy and coulter counting.

\section{Characterization of Acoustic Vesicles using Multisizer}

The acoustic vesicles were characterized using a Multisizer 4e Coulter Counter to determine the size distribution and concentration of formed acoustic vesicles. A fixed concentration of microcapsules was used for the preparation of acoustic vesicles and separated using the centrifugation technique mentioned above. Separated acoustic vesicles were used for characterization using coulter counting. This experiment was done in triplicate.

\section{Scanning Electron Microscopy}

Scanning electron microscopy (SEM) was done on two samples: 1) Separated acoustic vesicles 2) microcapsule pellet after centrifugation. To prepare the samples for SEM microscopy, acoustic vesicles were made using mentioned protocols. After gas nucleation, the sample went through 8 centrifugations at $1500 \times \mathrm{g}$ and the resulting pellet was used for microscopy as the control sample. The buoyant particles were collected after each centrifuge and were centrifuged three more times at $300 \times g$ and the resulting sample were used for SEM imaging as acoustic vesicles. The samples were transferred to a microscopic glass and a thin layer of gold/palladium ( $\mathrm{Au} / \mathrm{Pd})$ was sputter-coated on the sample. SEM images were acquired at $2.00 \mathrm{kV}$.

\section{Confocal Microscopy}

Olympus FV3000RS Confocal microscope was used for visualizing calcein-loaded acoustic vesicles. Calcein-loaded acoustic vesicles were produced as mentioned earlier and were separated by the centrifugation process. A diluted sample of acoustic vesicles was seen under a confocal microscope at excitation and emission wavelength of $490 \mathrm{~nm}$ and $520 \mathrm{~nm}$ respectively. Image software was used for processing the images and construction of 3D figures.

\section{Calcein Loading Measurements}

To measure the amount of loaded calcein in the acoustic vesicle sample, calcein-loaded acoustic vesicles were generated and separated based on the methods mentioned earlier. Dichloromethane was used for extracting calcein from acoustic vesicles. A fixed amount of calcein-loaded acoustic vesicles was first diluted by 1 XPBS to keep the PH steady and the diluted sample was added to dichloromethane. Acoustic vesicles were mixed with dichloromethane by vigorous hand mixing for $20 \mathrm{~s}$. After vigorous mixing, the sample sat for $1 \mathrm{~min}$ to phase separate and dichloromethane was extracted from the bottom of the vial using a glass syringe. Fresh dichloromethane was added to the sample and this step was repeated 10 times. After the extractions, the fluorescence intensity of the sample was measured by plate reading at $490 \mathrm{~nm}$ excitation and $520 \mathrm{~nm}$ emission wavelengths, and calcein concentration was calculated using a standard concentration curve. T-test was done on the dataset for statistical analysis.

\section{Acoustic Characterization}


A focused transducer with a center frequency of $1.1 \mathrm{MHz}$ was used as the excitation source. A focused hydrophone, confocally aligned with the source transducer at a 90-degree angle was used to passively acquire the cavitation signals. 500 cycle pulses with a pulse repetition frequency of 40 $\mathrm{Hz}$ were transmitted at peak-negative pressures ranging from $0.44 \mathrm{MPa}$ to $7 \mathrm{MPa}$. 100 echoes were collected at each pressure level. After transforming each to the frequency domain, the resulting power spectrum was detrended using a moving median filter to exclude the broadband noise component. The stable cavitation dosage (SCD) was defined as the sum of the area under curve (AUC) values at selected frequencies. For calculating this dosage, $\pm 10 \%$ bandwidth around the $1^{\text {st }}, 2^{\text {nd }}$ and subharmonic frequencies were selected from the detrended power spectrum and the corresponding AUC values were calculated. The final SCD at any time interval was taken as the average of the 100 SCD values. All data processing was done offline using custom LabVIEW software (LabVIEW 2015, National Instruments, Austin, TX, USA). Finally, SCD against ultrasound pressure was plotted and the area under each curve was calculated. An ANOVA test was done on the area under the curve measurements followed by a t-test between every two samples to measure significance.

\section{Acoustic Radiation Forces on Acoustic Vesicles}

It is known that bubbles can be displaced in a liquid medium by applying low-pressure acoustic waves. This is due to the absorption of the sound wave momentum ${ }^{2,3}$. This phenomenon is particularly advantageous in drug delivery applications by improving the local delivery of drugs ${ }^{4}$. A simple apparatus was designed to perfuse acoustic vesicles in a capillary tube and visualize the effect of low-intensity ultrasound on the acoustic vesicles (Figure S3). A sample of acoustic vesicles was perfused in a capillary tube right after nucleation. The microscope objective was aligned with the focal point of the transducer and a pulse train (one million total pulses at $1.1 \mathrm{MHz}$ ) of $0.18 \mathrm{MPa}$ ultrasound, similar to ultrasound parameters established in the literature ${ }^{5}$ was applied to the acoustic vesicles. The videos were taken from the top of the capillary tube to capture the acoustic vesicles and from the bottom of the capillary tube to visualize non-bubble-loaded microcapsules.

\section{Supplementary Figures}

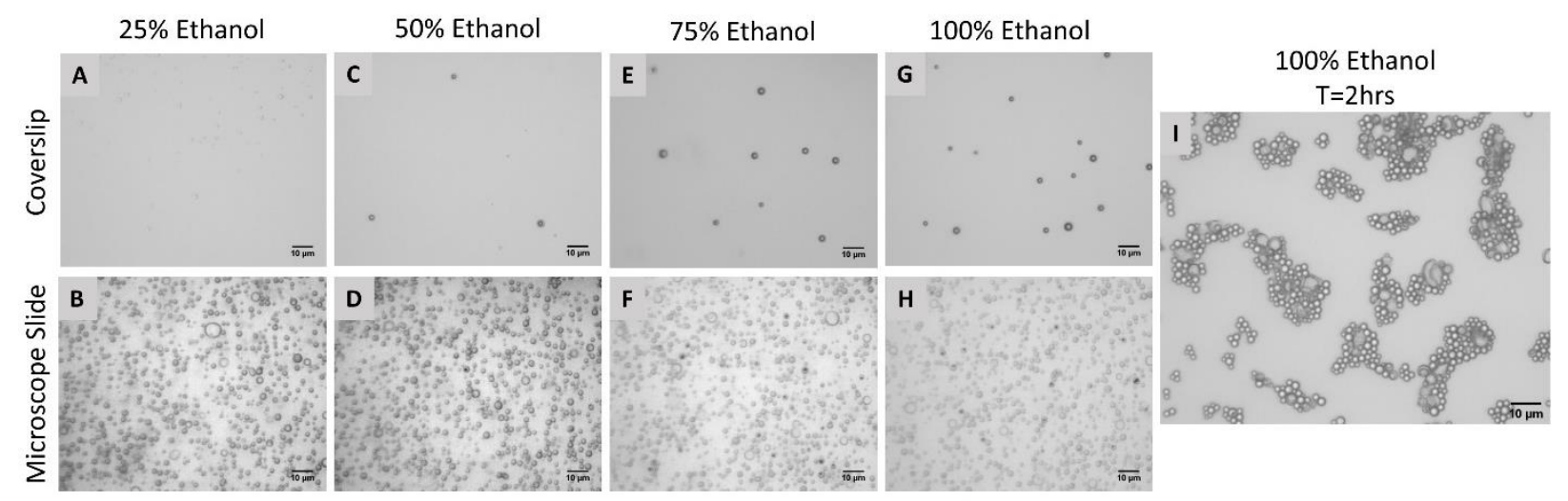

Figure S1. Using water and ethanol for generating acoustic vesicles. By adding water to the mixture of ethanol and PLA microcapsules, acoustic vesicles will form. By increasing the concentration of initial ethanol, the number of formed acoustic vesicles will increase. This is an indication that increasing the degree of supersaturation causes an improved yield of acoustic vesicle production. Buoyant bubbles will rise to the top while unloaded microcapsules or microcapsules with smaller bubbles will sink to the bottom. A-H) Bubble nucleation increases by increasing ethanol concentration. I) Aggregation of microcapsules over time in ethanol solution. 


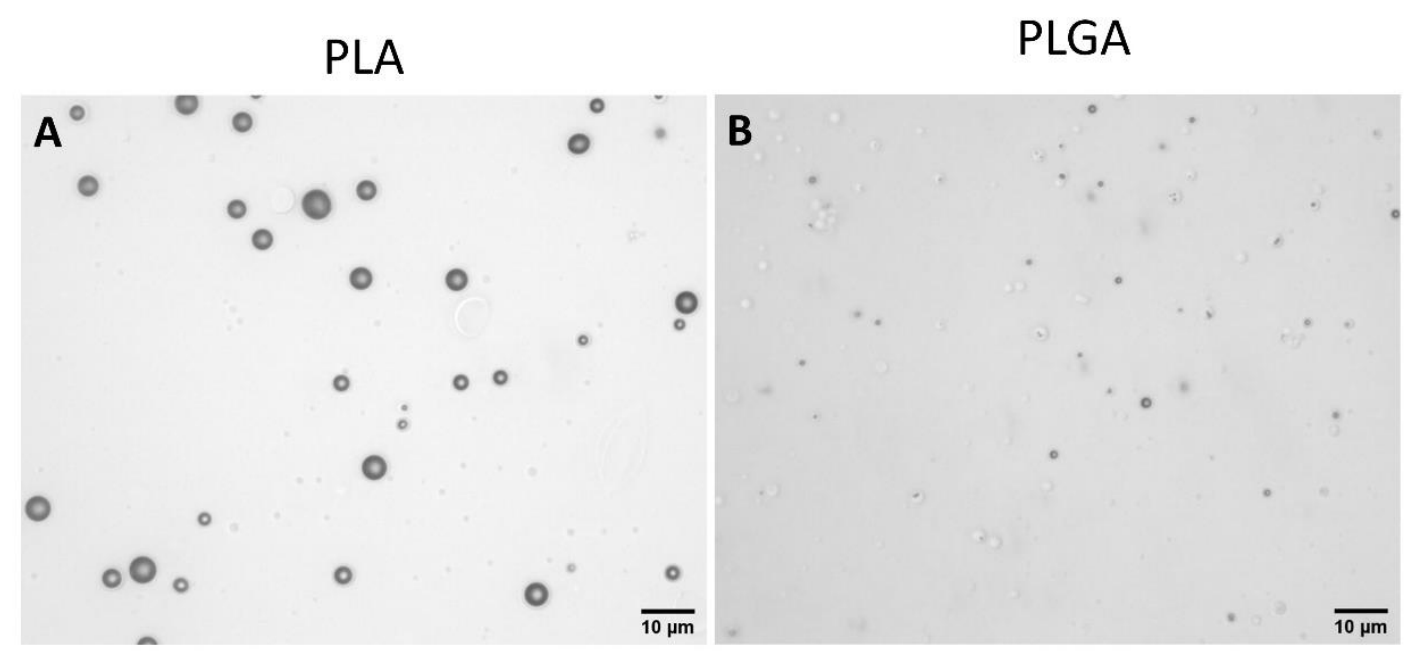

Figure S2. Production of poly lactic-co-glycolic (PLGA) acoustic vesicles. This image is from the coverslip showing the feasibility of producing acoustic vesicles from PLGA.

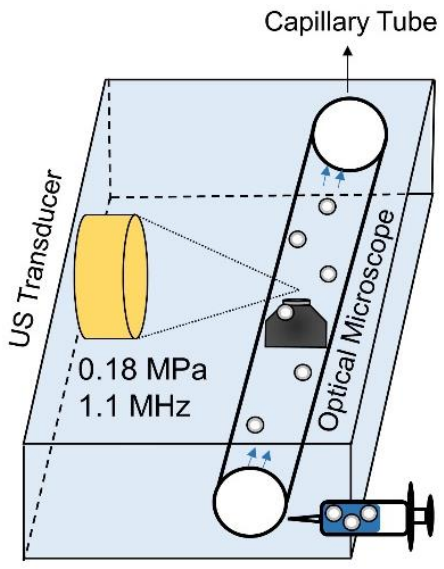

Figure S3. Schematic figure of the setup used for visualizing acoustic radiation force on acoustic vesicles. The microscope objective was co-aligned with the ultrasound transducer to capture the acoustic effects at the focal point.

\section{Supplementary Videos}

\section{Video S1. Nucleation in Microcapsules Embedded in Agarose Gel}

Supplementary Video 1a: This video is a time-lapse video of bubble nucleation in the microcapsules. Microcapsules are embedded in 1\% agarose and 30\% propylene glycol, $30 \%$ glycerol 1 XPBS is added to the gel, changing the air solubility. Bubbles nucleate as the solution slowly diffuses in the gel. This is a clear indication of bubble nucleation.

Supplementary Video 1b: As a control, we perfused 1XPBS to the perfusion chamber.

\section{Video S2. Radiation Force Applications in a Cellulose Capillary Tube}


In this experiment, an unwashed sample of acoustic vesicles was perfused in a capillary tube, and a single pulse train ( 1 million pulses total) of ultrasound was applied to the sample at $0.18 \mathrm{MPa}$ and 1.1 MHz.

Video S2a: As seen in the videos, the acoustic vesicles at the top of the capillary tube respond to ultrasound (at 1 second) and are pushed to the capillary tube wall by radiation forces.

Video S2b: The microcapsules at the bottom of the capillary tube are not responsive to ultrasound. This is proof that bubbles are present in the acoustic vesicles and can be displaced using low-intensity focused ultrasound.

\section{References}

(1) El-Sherif, D. M.; Wheatley, M. A. Development of a Novel Method for Synthesis of a Polymeric Ultrasound Contrast Agent. Journal of Biomedical Materials Research Part A 2003, 66A (2), 347-355. https://doi.org/10.1002/jbm.a.10586.

(2) Dayton, P.; Klibanov, A.; Brandenburger, G.; Ferrara, K. Acoustic Radiation Force in Vivo: A Mechanism to Assist Targeting of Microbubbles. Ultrasound Med Biol 1999, 25 (8), 1195-1201. https://doi.org/10.1016/s0301-5629(99)00062-9.

(3) Zhao, S.; Borden, M.; Bloch, S. H.; Kruse, D.; Ferrara, K. W.; Dayton, P. A. Radiation-Force Assisted Targeting Facilitates Ultrasonic Molecular Imaging. Mol Imaging 2004, 3 (3), 135-148. https://doi.org/10.1162/1535350042380317.

(4) Lum, A. F. H.; Borden, M. A.; Dayton, P. A.; Kruse, D. E.; Simon, S. I.; Ferrara, K. W. Ultrasound Radiation Force Enables Targeted Deposition of Model Drug Carriers Loaded on Microbubbles. Journal of Controlled Release 2006, 111 (1), 128-134. https://doi.org/10.1016/j.jconrel.2005.11.006.

(5) Zhao, S.; Kruse, D. E.; Ferrara, K. W.; Dayton, P. A. Acoustic Response from Adherent Targeted Contrast Agents. J Acoust Soc Am 2006, 120 (6), EL63-EL69. https://doi.org/10.1121/1.2364303. 